TRANSACTIONS OF THE

AMERICAN MATHEMATICAL SOCIETY

Volume 192, 1974

\title{
ANALYTIC EQUIVALENCE IN THE DISK ALGEBRA
}

\author{
BY
}

\author{
HUGH E. WARREN
}

\begin{abstract}
The notion of analytically equivalent domains can be extended from the complex plane to commutative Banach algebras with identity. In $C(X)$ a domain equivalent to the unit ball must have a boundary that is in a certain sense continuous. This paper shows that in the disk algebra "continuous" must be replaced with "analytic." These results set limits in the classical Riemann mapping theorem on how smoothly the mapping can respond to changes in the domain being mapped.
\end{abstract}

Introduction. Thirty years ago E.R. Lorch [5] laid out a framework for analytic function theory in commutative Banach algebras with identity. In the past decade B.W. Glickfeld has developed these ideas in several directions. The paper [2] touches on analytic equivalence, there called analytic isomorphism. The present paper concerns itself with domains in the disk algebra which are analytically equivalent to the unit ball. Previous papers by the author [6], [7] have considered this problem for algebras of the form $C(X)$. The results in both $C(X)$ and in the disk algebra are motivated by Lorch's definition of a simple closed curve.

Let $A$ be a commutative Banach algebra with identity and let $T$ be the unit circle in the plane. A simple closed curve in $A$ is a continuous map $\gamma: T \rightarrow A$ with the property that $\gamma(t)-\gamma(s)$ is invertible in $A$ whenever $t \neq s$. Let $X$ be the maximal ideal space of $A$. We see the reason for the term "simple" if, following Gel'fand, we represent $A$ as an algebra of continuous functions on $X$. Under this representation $\gamma$ maps each $t \in T$ to a function $\gamma[t]$ in $C(X)$. The value of $\gamma[t]$ at $z \in X$ is the complex number $\gamma[t](z)$. Let $E_{z}$ be the evaluation functional $E_{z}(f)=f(z)$ and put $\gamma_{z}=E_{z} \circ \gamma$. The function $\gamma_{z}$ is continuous because it is a composition of continuous functions. It is one-to-one because if $t \neq s$, then $\gamma[t]-\gamma[s]$ is invertible and $\gamma_{z}(t)-\gamma_{z}(s)=\gamma[t](z)-\gamma[s](z) \neq 0$. Geometrically $\gamma_{z}$ is a simple closed curve in the plane.

Given a simple closed curve $\gamma$ in $A$, we define its interior, int $\gamma$, and its enclosure, enc $\gamma$. Each curve $\gamma_{z}$ has an interior, int $\gamma_{z}$, in the usual sense and an enclosure, enc $\gamma_{2}$, which is the closure in the plane of the interior. We put

$$
\text { int } \gamma=\left\{f \in A: f(z) \in \text { int } \gamma_{z} \text {, all } z \in X\right\} \text {. }
$$

Define enc $\gamma$ by putting enc in place of int. We know very little about int $\gamma$ in general, not even whether it is empty. The potential of int $\gamma$ lies in the following result from [6].

Received by the editors March 21, 1972 and, in revised form, March 9, 1973.

AMS (MOS) subject classifications (1970). Primary 30A96, 46J15; Secondary 30A30.

Key words and phrases. Disk algebra, Lorch analytic function, analytic equivalence.

Copyright $\odot$ 1974, American Mathematical Society 
Theorem C. If $\gamma$ is a simple closed curve in $A=C(X)$, then there is a homeomorphism $\Phi$ of the closed unit ball onto enc $\gamma$ such that $\Phi$ is an analytic equivalence of the open unit ball onto int $\gamma$.

Returning to an arbitrary algebra $A$, let $U$ and $\bar{U}$ respectively be the open and closed unit ball. A simple closed curve $\gamma$ in $A$ will be called resolvable if there exists a homeomorphism $\Phi$ of $\bar{U}$ onto enc $\gamma$ which is an analytic equivalence of $U$ onto int $\gamma$. Theorem $C$ says that in $C(X)$ every simple closed curve is resolvable. This is not true in the disk algebra. The next section characterizes those curves in the disk algebra which are resolvable and gives examples of curves which are not.

It is important to note that when a resolution $\Phi$ does exist, it operates pointwise with respect to the maximal ideal space $X$ of the algebra $A$. Being analytic on $U$ gives $\Phi$ a power series representation $\Phi[f]=\sum g_{n} f^{n}$, where the coefficient functions $g_{n}$ belong to $A$. For each $z \in X$ we get a "quotient function" $\Phi_{z}(w)=\sum g_{n}(z) w^{n}$ defined on the unit disk. The analytic map $\Phi_{z}$ extends to a homeomorphism of the closed unit disk onto enc $\gamma_{z}[4$, p. 367]. The pointwise behavior of $\Phi$ is summed up in the equation $\Phi[f](z)=\Phi_{z}(f(z))$. For more on quotient functions see [2].

The third section of this paper shows that for certain curves the interior is analytically equivalent to the unit ball only if the curve is resolvable. The final section discusses curves in the disk algebra in relation to the classical Riemann mapping theorem.

I am much indebted to the referee for his advice and assistance.

Curves in the disk algebra. In the complex plane let $K$ and $K$ respectively be the open and closed unit disk. The disk algebra $D$ is the subalgebra in $C(K)$ of functions analytic on $K$. The norm in $D$ is the uniform norm over $R$. Functions in $D$ are subject to the maximum principle, which is reflected in the following result.

Theorem 1. If $\gamma$ is a simple closed curve in $D$ and $f \in$ enc $\gamma$, then either $f=\gamma[t]$ for some $t \in T$ or else $f(z) \in$ int $\gamma_{z}$ for all $z \in K$.

Proof. The assumption $f \in$ enc $\gamma$ means that for every $z$ the value $f(z)$ lies either inside or on $\gamma_{z}(T)$. If it lies on $\gamma_{z}(T)$, then $f(z)=\gamma[t](z)$ for some $t \in T$. Suppose $f \neq \gamma\left[t_{0}\right]$ and $z_{0} \in K$. We shall show that $f\left(z_{0}\right) \neq \gamma\left[t_{0}\right]\left(z_{0}\right)$. Letting $t_{0}$ and $z_{0}$ vary, we get the theorem as stated.

The functions $f$ and $\gamma\left[t_{0}\right]$ are analytic near $z_{0}$. Since $f$ and $\gamma\left[t_{0}\right]$ are unequal, their difference does not vanish on some small circle $S$ centered at $z_{0}$ and lying in $K$. For $t \neq t_{0}$ the analytic function $\gamma[t]-\gamma\left[t_{0}\right]$ has no zeros at all because $\gamma$ is a simple curve. It follows that the winding number of $\gamma[t]-\gamma\left[t_{0}\right]$ on $S$ is zero. The winding number of $f-\gamma\left[t_{0}\right]$ on $S$ must also be zero because, roughly speaking, the value of $f-\gamma\left[t_{0}\right]$ at any point of $S$ is caught in the curve of values of $\gamma[t]-\gamma\left[t_{0}\right]$ as $t$ varies over $T$. An appeal to Theorem $C$ will make this precise. 
Let $\gamma_{S}[t]$ and $f_{S}$ be the restrictions to $S$ of $\gamma[t]$ and $f$. One checks that $\gamma_{S}$ is a simple closed curve in $C(S)$ and that $f_{S} \in$ enc $\gamma_{S}$. Let $\Phi$ be the homeomorphism of Theorem C. Choose $t_{1} \in T$ with $t_{1} \neq t_{0}$ and let $g_{0}, g_{1}, f_{1}$ be the preimages under $\Phi$ of $\gamma_{s}\left[t_{0}\right], \gamma_{s}\left[t_{1}\right], f_{S}$ respectively. From the pointwise property of $\Phi$ one sees that $g_{0}$ and $g_{1}$ take unequal values on $T ; f_{1}$ takes values in $\bar{K}$ and never equals $g_{0}$, because $f$ and $\gamma\left[t_{0}\right]$ are never equal on $S$. Look at the path of functions $(1-x) f_{1}+x g_{1}, 0 \leq x \leq 1$. Under $\Phi$ this path is mapped to a homotopy from $f_{S}$ to $\gamma_{S}\left[t_{1}\right]$ such that no function in the homotopy ever agrees with $\gamma_{S}\left[t_{0}\right]$. The existence of this homotopy shows that $f-\gamma\left[t_{0}\right]$ and $\gamma\left[t_{1}\right]-\gamma\left[t_{0}\right]$ have the same winding number on $S$, namely zero. By the argument principle $f-\gamma\left[t_{0}\right]$ does not vanish inside $S$, in particular $f\left(z_{0}\right) \neq \gamma\left[t_{0}\right]\left(z_{0}\right)$.

The relation to the maximum principle is this. If $c[t]=t$ is the curve of constant functions of modulus one, then $|f(z)| \leq 1$ for $z \in R$ is equivalent to $f \in$ enc $c$. By the maximum principle $|f(z)| \leq 1$ for $z \in \bar{K}$ implies either $f$ is a constant of modulus one, that is, $f=c\left[t_{0}\right]$ for some $t_{0}$, or $|f(z)|<1$, that is, $f(z) \in$ int $c_{z}$, for all $z \in K$.

The unit circle $T$ plays a dual role for a curve in the disk algebra. It is the domain of the curve, and it is the boundary of the disk $\bar{K}$. For resolvable curves these roles are connected.

Theorem 2. $A$ resolvable curve $\gamma$ in $D$ can be reparameterized so that every $\gamma_{z}$ extends to a function in $D$.

Proof. Let $\gamma$ be the resolvable curve in $D$ and let $\Phi$ be a mapping that resolves $\gamma$. Think of $T$ lying in $\bar{U}$ as the constant functions of modulus one. Let $\gamma^{*}$ be the restriction to $T$ of $\Phi$. The pointwise property of $\Phi$ shows that $\gamma^{*}$ is a simple closed curve and that $\gamma^{*}[t](z)$ lies on $\gamma_{z}(T)$ for all $z \in \bar{K}$. By Theorem 1 each $\gamma^{*}[t]$ is equal to some $\gamma\left[t^{\prime}\right]$. Since $\gamma^{*}$ and $\gamma$ are simple closed curves, every $\gamma\left[t^{\prime}\right]$ is some $\gamma^{*}[t]$. In other words $\gamma^{*}$ is a reparameterization of $\gamma$. The extension of $\gamma_{z}^{*}$ to a function in $D$ is the quotient function $\Phi_{2}$.

Simple closed curves with the property that each $\gamma_{z}$ extends to a function in $D$ will be called $D$-curves.

Theorem 3. A D-curve in the disk algebra is resolvable.

Proof. Let $\gamma$ be the $D$-curve and consider the functions $\gamma_{z}$ to be already extended to functions in $D$. Since $\gamma_{z}$ is one-to-one on $T$, the argument principle shows that $\gamma_{z}$ is also one-to-one on $\bar{K}$. Moreover $\gamma_{z}$ maps $\bar{K}$ onto enc $\gamma_{z}$ and $K$ onto int $\gamma_{z}$.

For $f \in \bar{U}$ and $g \in$ enc $\gamma$ define functions $\Phi[f]$ and $\Psi[g]$ by

$$
\Phi[f](z)=\gamma_{z}(f(z)) \text { and } \Psi[g](z)=\gamma_{z}^{-1}(g(z)) \text {. }
$$

These functions are well defined because $f(z) \in \bar{K}$ and $g(z) \in$ enc $\gamma_{z}$. They are also bounded, $\Phi[f]$ by $\max \|\gamma[t]\|$ and $\Psi[g]$ by 1 . Thus $\Phi$ and $\Psi$ are mappings into 
$B(\bar{K})$, the space of bounded functions on $K$. To discuss the continuity of $\Phi$ and $\Psi$ we give $B(\bar{K})$ the uniform norm.

We shall show that $\Phi$ resolves $\gamma$. Once we know that $\Phi$ and $\Psi$ map into $D$, it will be clear that they are inverse to one another. We first consider the action of $\Phi$ and $\Psi$ on $U$ and int $\gamma$. For $f \in U$ the Cauchy integral

$$
(2 \pi i)^{-1} \int_{T}(t-f)^{-1} \gamma[t] d t
$$

defines a function in $D$. (It is a uniform limit of Riemann sums in $D$.) The value of this function at $z$ is

$$
(2 \pi i)^{-1} \int_{T}(t-f(z))^{-1} \gamma_{z}(t) d t=\gamma_{z}(f(z))=\Phi[f](z) .
$$

We can interchange evaluation and integration because evaluation is a continuous functional. The subsequent equality holds because $\gamma_{z} \in D$. Thus $\Phi$ is given by a Cauchy integral and is analytic on $U$. Since $\gamma_{z}(K)=$ int $\gamma, \Phi$ maps $U$ into int $\gamma$.

If $f \in \bar{U}$ is a constant function $f(z) \equiv w$, we can replace the Cauchy kernel in (1) with the Poisson kernel and obtain a function $\gamma[w]$ in $D$. Since $\gamma$ is continuous on $T$, this procedure makes $\gamma$ a continuous function on $\bar{K}$. The value of $\gamma[w]$ at $z$ is $\gamma_{z}(w)$, because for functions in $D$ the Cauchy and Poisson integrals agree. For $w \in K$ the derivative $\gamma^{\prime}[w]$ belongs to $D$ and satisfies $\gamma^{\prime}[w](z)=\gamma_{z}^{\prime}(w)$.

Suppose $g \in$ int $\gamma$. The compactness of $R$ implies there exists $r<1$ such that $g(z)$ lies inside the curve $\gamma_{z}(r T)$ for all $z \in R$. The Cauchy integral

$$
(2 \pi i)^{-1} \int_{r T}(\gamma[t]-g)^{-1} \gamma^{\prime}[t] t d t
$$

represents a function in $D$. The contour of integration is $r T$ and the integral is unchanged if we increase $r$ towards 1 . We must, however, keep $r<1$ so that $\gamma^{\prime}[t]$ exists. Evaluating (2) at $z$, we get

$$
(2 \pi i)^{-1} \int_{r T}\left(\gamma_{z}(t)-g(z)\right)^{-1} \gamma_{z}^{\prime}(t) t d t=\gamma_{z}^{-1}(g(z))=\Psi[g](z) .
$$

We see that $\Psi$ is analytic on int $\gamma$ and maps int $\gamma$ into $U$. Consequently $\Phi$ is an analytic equivalence of $U$ onto int $\gamma$ with inverse $\Psi$.

We next show that $\Phi$ and $\Psi$ are continuous as mappings into $B(R)$. The map $\gamma$ is uniformly continuous on the compact set $R$, say $|u-v|<\delta(\varepsilon)$ implies $\|\gamma[u]-\gamma[v]\|<\varepsilon$. If $f$ and $g$ in $U$ satisfy $\|f-g\|<\delta(\varepsilon)$, then $|f(z)-g(z)|$ $<\delta(\varepsilon)$. From the formula $\Phi[f](z)=\gamma[f(z)](z)$ we see that

$$
\begin{aligned}
|\Phi[f](z)-\Phi[g](z)| & =|\gamma[f(z)](z)-\gamma[g(z)](z)| \\
& \leq\|\gamma[f(z)]-\gamma[g(z)]\|<\varepsilon .
\end{aligned}
$$

Taking the maximum over $z \in \bar{K}$, we get $\|\Phi[f]-\Phi[g]\|<\varepsilon$ and conclude that $\Phi$ is uniformly continuous on $\bar{U}$. Being continuous, $\Phi$ sends convergent sequenc- 
es in $\bar{U}$ to convergent sequences in $B(\bar{K})$. We know that $U$ is the closure of $U$, that $\Phi$ maps $U$ into $D$ and that $D$ is closed in $B(R)$. It follows that $\Phi$ maps $U$ into $D$.

Now to the continuity of $\Psi$. The quantity $q(z, u, v)=\left|\gamma_{z}(u)-\gamma_{z}(v)\right|$ is a continuous function of $(z, u, v)$ in $\bar{K} \times \bar{R} \times \bar{K}$, and $q$ vanishes only when $u=v$. For $\varepsilon>0$ the subset of $\bar{K} \times \bar{K} \times \bar{K}$ on which $|u-v| \geq \varepsilon$ is compact, and $q>0$ on it; therefore $q$ has a positive minimum $m(\varepsilon)$ on this set. Suppose $f$ and $g$ belong to enc $\gamma$ with $\|f-g\|<m(\varepsilon)$. Put $u=\Psi[f](z)$ and $v=\Psi[g](z)$. From the definition of $\Psi$ we get $q(z, u, v)<m(\varepsilon)$, hence

$$
|\Psi[f](z)-\Psi[g](z)|=|u-v|<\varepsilon .
$$

Taking the maximum over $z \in K$ we get $\|\Psi[f]-\Psi[g]\|<\varepsilon$ and conclude that $\Psi$ is uniformly continuous on enc $\gamma$. Arguing as with $\Phi$, we show $\Psi$ maps the closure of int $\gamma$ into $D$. We have not shown that the closure of int $\gamma$ is actually enc $\gamma$.

Fix $g \in$ enc $\gamma$. For the moment think of $\gamma$ as a curve in $C(R)$. All of the proof so far goes through with $C(K)$ in place of $D$. Moreover Theorem $C$ tells us that in $C(\bar{K})$ the enclosure is the closure of the interior. Thus $\Psi[g]$ is at least continuous on $\bar{K}$. If $g=\gamma[t]$ for some $t$, then the definition of $\Psi$ shows that $\Psi[g]$ is the constant $t$. Suppose $g$ is not one of the $\gamma[t]$. Theorem 1 says that $g(z) \in$ int $\gamma_{z}$ for all $z \in K$. Fix $z_{0} \in K$. The continuity of $g$ and $\gamma$ gives us $r<1$ and $\varepsilon>0$ so that $\left|z-z_{0}\right|<\varepsilon$ implies $g(z)$ lies inside $\gamma_{z}(r T)$. Thus for $\left|z-z_{0}\right|$ $<\varepsilon$ we can evaluate the integral (2) at $z$ and obtain equation (3). Since $\gamma[t], g$ and $\gamma^{\prime}[t]$ are analytic at $z_{0}$, so is $\Psi[g]$. Letting $z_{0}$ vary over $K$ shows $\Psi[g]$ is analytic on $K$, that is, $\Psi[g] \in D$.

We have shown that $\Phi$ and $\Psi$ map into $D$. We see from their definitions they can be composed in either order and are inverse to each other. They are appropriately continuous and analytic, and the proof is done.

Since reparameterization does not change the interior or enclosure of a curve, the preceding results say that in the disk algebra the resolvable curves are precisely the $D$-curves and their reparameterizations. To construct examples a further characterization is convenient.

Lemma. If $\gamma$ is a simple closed curve in $D$ and $z$ is a point in $R$, then $\gamma$ can be reparameterized so that $\gamma_{z}$ extends to a function in $D$.

Proof. Use the classical Riemann mapping theorem to produce an analytic equivalence $f$ of $K$ onto int $\gamma_{z}$. It is known that $f$ extends to a homeomorphism of $\bar{K}$ onto enc $\gamma_{z}[4$, p. 367]. In particular $f$ belongs to $D$. The desired reparameterization is $\gamma^{*}=\left.\gamma \circ \gamma_{z}^{-1} \circ f\right|_{r}$. The extension of $\gamma_{z}^{*}$ to a function in $D$ is $f$.

Theorem 4. If $\gamma$ is a simple closed curve in $D$ and $z$ a point in $R$, then $\gamma$ is resolvable if and only if any reparameterization which makes $\gamma_{z}$ extendable to a function in D simultaneously makes $\gamma$ a $D$-curve. 
Proof. If a reparameterization makes $\gamma$ a $D$-curve then by results above $\gamma$ is resolvable. Conversely let $\gamma$ be resolvable and suppose $\gamma$ has been reparameterized so that $\gamma_{z}$ extends to a function in $D$. Let $\gamma^{*}$ be the $D$-curve reparameterization induced by the resolution of $\gamma$ (as in Theorem 2). As functions in $D$ both $\gamma_{z}$ and $\gamma_{z}^{*}$ map $\bar{K}$ one-to-one onto enc $\gamma_{z}$. The function $f=\left(\gamma_{z}^{*}\right)^{-1} \circ \gamma_{z}$ is an analytic equivalence of $\bar{K}$ onto itself. One checks that for all $w \in \bar{K}$ we have $\gamma_{w}=\gamma_{w}^{*} \circ f$. Since $\gamma_{w}^{*}$ is a function in $D$ so is $\gamma_{w}$. In other words $\gamma$ is a $D$-curve.

We now construct an example of a simple closed curve in $D$ which is not resolvable. We call the curve $j$, and define it by $j[t](z)=2 t+\bar{t} z$ where $t \in T$ and $z \in R$. Each $j[t]$ is a linear function and clearly in $D$. The mapping $j: T \rightarrow D$ is continuous because $\left\|j\left[t_{1}\right]-j\left[t_{2}\right]\right\|=3\left|t_{1}-t_{2}\right|$. Writing $t=e^{i \theta}, r=|z|$ and $z=r e^{i \phi}$, we have

$$
j[t](z)=e^{i \phi / 2}\left\{(2+r) \cos \left(\theta-\frac{1}{2} \phi\right)+i(2-r) \sin \left(\theta-\frac{1}{2} \phi\right)\right\} .
$$

This formula shows that $j_{z}(t)=j[t](z)$ is one-to-one, that is, $j$ is simple. We can also see that $j_{2}(T)$ is an ellipse centered at the origin. The major and minor axes have lengths $2+r$ and $2-r$, and the major axis is rotated $\phi / 2$ radians from the horizontal. Theorem 4 shows that $j$ is not resolvable, for of all the $j_{z}$ only $j_{0}$ belongs to $D$. Note the $\bar{t}$ term.

As an example of a different type define $k\left[e^{i \theta}\right]$ to be piecewise linear in $\theta$ so that $k[ \pm 1]$ is the constant function \pm 1 respectively and $k[i](z)=2 i+z$. Each $k_{z}(T)$ is the triangle with vertices $-1,1$ and $2 i+z$. If $k$ were resolvable we would have a $D$-curve reparameterization $k^{*}$. Since $k$ maps an arc of $T$ to the constant functions between -1 and $1, k^{*}$ must map an arc $A$ to these same constant functions. Recall that $k_{z}^{*}(t)=k^{*}[t](z)$. For $t \in A$ and arbitrary $z, w \in R$ it follows that $k_{z}^{*}(t)=k_{w}^{*}(t)$. Since $k_{z}^{*}$ and $k_{w}^{*}$ belong to $D$, it must be that $k_{z}^{*}=k_{w}^{*}$ $\left[4\right.$, p. 365]. But if $k^{*}\left[t_{0}\right]=k[i]$, then $k_{z}^{*}\left(t_{0}\right)=k_{w}^{*}\left(t_{0}\right)$ only if $z=w$. This contradiction shows $k$ is not resolvable.

Interiors of curves. Resolvability is more than a condition on the interior of a curve. It is reasonable to ask if the interior of a curve such as $j$ or $k$ is analytically equivalent to $U$ even though such an equivalence could not extend to $U$ so as to make the curve resolvable. The answer is no, as we presently show.

Recall the evaluation functional $E_{z}(f)=f(z)$. Say that a simple closed curve $\gamma$ is full if $E_{z}$ (int $\left.\gamma\right)=$ int $\gamma_{z}$ for all $z$.

Theorem 5. Let $\gamma$ be a full simple closed curve. Every analytic equivalence of $U$ onto int $\gamma$ extends to a resolution of $\gamma$.

Proof. Let $\Phi$ be an analytic equivalence of $U$ onto int $\gamma$. In the terminology of [7] the domain int $\gamma$ is sectionally disk equivalent. By Lemma 4 of [7] the quotient function $\Phi_{z}$ is an analytic equivalence of $K$ onto int $\gamma_{z}=E_{z}$ (int $\gamma$ ). Although this result of [7] is stated for the algebra $C(X)$, the proof is the same for any commutative Banach algebra with identity. Since each $\gamma_{z}(T)$ is a Jordan curve, 
each $\Phi_{z}$ extends to a homeomorphism of $\bar{K}$ onto enc $\gamma_{z}$. We define $\Phi$ on $\bar{U}$ by $\Phi[f](z)=\Phi_{z}(f(z))$. This agrees with the pointwise property of $\Phi$ on $U$, and defines $\Phi[f]$ as a bounded function on $\bar{K}$.

We show that $\Phi: \bar{U} \rightarrow B(\bar{K})$ is continuous. We may assume that $\Phi[0]=0$ and $\Phi^{\prime}[0]=1$. For otherwise translate the whole problem by $-\Phi[0]$ and then multiply everything by the reciprocal of the nonvanishing function $\Phi^{\prime}[0]$ (nonvanishing because $\Phi$ is an analytic equivalence on $U$ ). These normalizations give us $\Phi_{z}(0)=0$ and $\Phi_{z}^{\prime}(0)=1$ for all $z$. Using the continuity of $\gamma$, one applies a theorem of Rado [3, p. 59] and shows that $\Phi_{z}$ converges to $\Phi_{w}$ uniformly on $R$ whenever $z$ converges to $w$. In other words, the mapping of $\bar{K}$ into $D$ given by $z \rightarrow \Phi_{z}$ is continuous. Since $\bar{K}$ is compact, the $\Phi_{z}$ form an equicontinuous family. For $\varepsilon>0$ there exists $\delta(\varepsilon)>0$ such that $|u-v|<\delta(\varepsilon)$ implies $\left|\Phi_{z}(u)-\Phi_{z}(v)\right|$ $<\varepsilon$ whenever $u, v$ and $z$ belong to $\bar{K}$. Now if $f$ and $g$ in $\bar{U}$ satisfy $\|f-g\|<\delta(\varepsilon)$, then $|f(z)-g(z)|<\delta(\varepsilon)$ and

$$
|\Phi[f](z)-\Phi[g](z)|=\left|\Phi_{z}(f(z))-\Phi_{z}(g(z))\right|<\varepsilon .
$$

Taking the maximum over $z \in \bar{K}$, we get $\|\Phi[f]-\Phi[g]\|<\varepsilon$ and conclude that $\Phi$ is continuous.

One shows as in the proof of Theorem 3 that $\Phi$ maps $\bar{U}$ into enc $\gamma$. Moreover, the pointwise property of $\Phi$ implies that $\Phi[t](z) \in \gamma_{z}(T)$ for all $t \in T$. By Theorem 1 each $\Phi[t]$ coincides with some $\gamma\left[t^{\prime}\right]$; indeed $\Phi$ restricted to $T$ is a $D$ curve reparameterization of $\gamma$. Theorem 3 and its proof show that $\Phi$ resolves $\gamma$.

The curves $j$ and $k$ are full. In fact let $\gamma$ be any simple closed curve such that all the sets enc $\gamma_{z}$ are convex. Fix $f \in$ int $\gamma$. Given $z \in \bar{K}$ and $u \in$ int $\gamma_{z}$, we have $t \in T$ and $x \in[0,1)$ so that $u=(1-x) f(z)+x \gamma[t](z)$. If $g=(1-x) f$ $+x \gamma[t]$, then $g \in$ int $\gamma$, and $g(z)=u$. Letting $u$ vary over int $\gamma_{z}$, we show that $E_{z}($ int $\gamma)=$ int $\gamma_{z}$.

The classical mapping problem. Given an embedding of the circle into the plane, we can construct an analytic equivalence of the unit disk onto the interior of the embedded curve. If the embedding is well behaved, then "construct" can be interpreted even in the strict sense of Bishop. In [1] Bishop structures the wellknown Koebe proof of the Riemann mapping theorem to his requirements. We cannot modify this explicit proof (or any other) to construct an analytic equivalence of the unit ball onto the interior of the curve $j$ in the disk algebra. What goes wrong first?

The Koebe proof starts with a simply connected domain $V$ normalized so that $0 \in V \subset K$. It then constructs successive conformal mappings of $V$ into $K$ which fix 0 and gradually pull the boundary of $V$ out to the unit circle. At each stage one pulls towards a point $c$ in $K-V$. Oddly enough it is choosing $c$ that will not generalize to the disk algebra.

Try the Koebe proof on int $j$. To mimic the normalization of $V$, scale down $j$ to $j / 3$. We have $0 \in$ int $j / 3 \subset U$, the unit ball $U$ being the analog of the unit 
disk $K$. There is no $g \in U$ with $g(z) \notin$ int $j_{z} / 3$ for all $z \in R$. For suppose $g \in U$ and $g(1) \notin$ int $j_{1} / 3$. Visualize the point $g(t)$ and the ellipse $j_{t}(t) / 3$ as $t$ takes one turn around $T$. The major axis of the ellipse, which is a diameter of the unit disk, makes one half turn. Note the $\phi / 2$ in (4). If $g(t)$ were never in int $j_{t} / 3$, then $g(t)$ would be swept from one side of the real axis to the other. That is impossible because $g$ is continuous.

Roughly speaking, we can construct the point $c$ above, but we cannot construct $c$ as a continuous function of the domain $V$. Ultimately curves such as $j$ and $k$ show that we cannot express a Riemann mapping of the disk onto a domain as an analytic function of the domain. For example, let $f_{z}$ be a suitably normalized Riemann mapping of $K$ onto int $k_{2}$. The vertices of $k_{z}(T)$, namely \pm 1 and $2 i+z$, are analytic functions of $z$, and one might expect the same for the coefficients in the power series of $f_{z}$. But they are not analytic, because $k$ is not resolvable. The coefficients are, however, continuous as can be seen from Theorem $\mathbf{C}$ or an application of the Schwarz-Christoffel formula to the polygonal curves $k_{z}$.

\section{REFERENCES}

1. E. Bishop, Foundations of constructive analysis, McGraw-Hill, New York, 1967, p. 112. MR 36 \#4930.

2. B. W. Glickfeld, On the inverse function theorem in commutative Banach algebras, Illinois J. Math. 15 (1971), 212-221. MR 42 \#8287.

3. G. M. Goluzin, Geometric theory of functions of a complex variable, GITTL, Moscow, 1952; English transl., Transl. Math. Monographs, vol. 26, Amer. Math. Soc., Providence, R.I., 1969. MR 15, 112; MR 40 \#308.

4. E. Hille, Analytic function theory. Vol. II, Introductions to Higher Math., Ginn, Boston, Mass., 1962. MR 34 \# 1490.

5. E. R. Lorch, The theory of analytic functions in normed abelian vector rings, Trans. Amer. Math. Soc. 54 (1943), 414-425. MR 5, 100.

6. H. E. Warren, A Riemann mapping theorem for $C(X)$, Proc. Amer. Math. Soc. 28 (1971), 147-154. MR 43 \#5300.

7.—, Sets in $C(X)$ analytically equivalent to the open ball, Duke Math. J. 39 (1972), 711-717.

Department of Mathematics, Universtty of Arizona, Tucson, Arizona 85721 